Patrick Charaudeau. 2020. La manipulation de la vérité. Du triomphe de la négation aux brouillages de la post-vérité (Limoges : Lambert-Lucas)

\title{
Sébastien Chonavey
}

\section{(2) OpenEdition}

Journals

Electronic version

URL: http://journals.openedition.org/aad/5367

DOI: 10.4000/aad.5367

ISSN: 1565-8961

Publisher

Université de Tel-Aviv

\section{Electronic reference}

Sébastien Chonavey, "Patrick Charaudeau. 2020. La manipulation de la vérité. Du triomphe de la négation aux brouillages de la post-vérité (Limoges : Lambert-Lucas)", Argumentation et Analyse du Discours [Online], 26 | 2021, Online since 14 April 2021, connection on 16 April 2021. URL: http:// journals.openedition.org/aad/5367 ; DOI: https://doi.org/10.4000/aad.5367

This text was automatically generated on 16 April 2021.

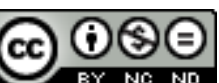

Argumentation \& analyse du discours est mis à disposition selon les termes de la licence Creative Commons Attribution - Pas d'Utilisation Commerciale - Pas de Modification 4.0 International. 


\title{
Patrick Charaudeau. 2020. La manipulation de la vérité. Du triomphe de la négation aux brouillages de la post-vérité (Limoges : Lambert- Lucas)
}

\author{
Sébastien Chonavey
}

\section{REFERENCES}

Patrick Charaudeau. 2020. La manipulation de la vérité. Du triomphe de la négation aux brouillages de la post-vérité (Limoges : Lambert-Lucas), 172 p., ISBN 978-2-35935-316-7

L'ouvrage de Patrick Charaudeau édité aux éditions Lambert-Lucas, et pertinemment illustré par Xavier Gorce, met en évidence les apports fertiles d'une approche linguistique de la manipulation en s'interrogeant sur ses conditions d'analyse et sur ses rapports avec le phénomène de la post-vérité. L'ouvrage synthétise les réflexions d'un analyste du discours réputé, chercheur au laboratoire de communication et politique du CNRS et professeur émérite de l'Université Paris XIII. D'emblée, l'auteur choisit de caractériser la manipulation de la vérité comme une forme de communication qui rompt avec les principes de pertinence et d'altérité. En effet, d'un côté, le sujet manipulateur est « mû par une négativité vis-à-vis des différentes formes de vérité »; de l'autre, le manipulé est considéré comme un être dénué de réaction critique à l'encontre de ce qu'affirme le manipulateur. Ce faisant, Charaudeau propose de recadrer l'analyse de ce type de discours sur la responsabilité et le langage des sujets ("responsabilité du manipulateur dans son vouloir-tromper » et "du manipulé dans son vouloir se soumettre », 161) plutôt que sur celui, plus abstrait, de la société (on songe par exemple à Ellul 1962 ou Huyghe 2016). De là, une tentative de proposer un examen centré sur les locuteurs (et les allocutaires) d'un phénomène marquant de 
notre époque, notamment depuis l'enregistrement du mot post-truth et son élection, en 2016, comme mot de l'année par le dictionnaire d'Oxford.

Fidèle à la tradition scientifique selon laquelle à mesure que l'on devient compétent, on teinte son assurance d'une plus grande prudence (Klein 2020 : 18), Charaudeau prend le parti de regrouper des interprétations qu'il sait provisoires et éphémères du fait du mouvement éternel de la recherche. Centrées autour de la problématique du discours manipulatoire (9), ses réflexions se proposent d'apporter un éclairage supplémentaire sur une forme d'utilisation du langage liée à de nombreux faits d'actualité. Cette perspective semble d'autant plus précieuse que, comme le signale l'auteur dans sa conclusion, cette thématique s'inscrit, pour nos sociétés, dans un temps de crises (149) - crises de la communication, de la vérité, du savoir ou de la confiance (politique notamment). L'ouvrage puise, pour étayer ses observations, dans un corpus très large, regroupant à la fois des citations célèbres, des faits historiques, des raisonnements logiques... De là, une analyse conceptuelle ambitieuse soutenue par de nombreux exemples empruntés à l'actualité récente mais aussi, en ce qui concerne la contextualisation de la post-vérité, à des faits beaucoup plus anciens comme les rumeurs anti-juives au Moyen-Âge ou celles entourant la Bête de Gévaudan au 18e siècle.

Pour mener à bien son entreprise, Charaudeau a choisi de conduire son exploration sur l'axe du langage en raison de son importance: il accompagne l'homme depuis sa naissance et lui permet de donner une vision du monde tout en instituant des rapports de force dans une société. Cependant, cette préférence pour l'analyse linguistique n'empêche pas l'auteur de faire précéder ses réflexions, dans un souci explicite d'interdisciplinarité, de clarifications extraites de nombreuses références philosophiques permettant de situer plus clairement les assises épistémologiques de son travail. Cette démarche marque l'apport spécifique du livre par rapport à d'autres ouvrages importants sur la question du rapport de nos sociétés à la vérité. $\mathrm{Si}$, comme le montre Gérald Bronner (2020), la confrontation de nos invariants cognitifs avec le numérique et la libéralisation du marché de l'information révèle bien une nouvelle "apocalypse cognitive", Charaudeau souligne également l'importance des jeux interactionnels de persuasion dont il s'agit, selon lui, de comprendre la tension entre savoirs de connaissance (28) et savoirs de croyance (31). De même, suivant l'approche journalistique de Laurent Bigot sur les apports de la vérification (2019) dans le processus journalistique de diffusion des nouvelles, l'auteur insiste sur le fait que les fake-news sont elles-mêmes le fruit d'une construction prise entre deux éléments. D'un côté, les injonctions contradictoires des médias (par exemple la déontologique neutralité de jugement opposée à l'engagement critique que suppose l'art du décryptage), de l'autre, le triomphe de la négativité (146) exprimé par le désir paradoxal de ne pas se laisser tromper et en même temps se méfier de la vérité (Williams 2006). En cela, Charaudeau rejoint une partie des analyses de Mauricio Ferraris sur l'émergence de la post-vérité comme inflation de la postmodernité (Ferraris 2019: 13) qui traduirait une forme d'indifférence à la vérité devenue « inessentielle» (Charaudeau, 154). L'auteur ne manque pas cependant de complexifier plus encore cette problématique en pointant notamment les diverses questions d'intentionnalité et d'intersubjectivité que suppose l'approche de la vérité par le langage.

4 En effet, afin de pouvoir procéder à une analyse détaillée des actes de langage manipulatoires, l'auteur se propose d'abord de défricher les rapports possibles du sujet 
à la vérité. Familier de ces questions (Charaudeau 2007), il apporte un certain nombre d'outils conceptuels permettant de travailler sur la problématique du rapport à la vérité. Traitant ainsi de la relation de la vérité aux types de connaissances, il suggère de distinguer quatre types de savoirs également répartis entre "savoirs de connaissance " et «savoirs de croyance ». D'un côté, les « savoirs de connaissance » résulteraient de représentations construites par le langage pour s'instituer en savoir et tendraient «à établir une vérité qui porte sur l'existence des faits du monde et qui propose des explications sur les phénomènes qui s'y produisent» (28). À l'intérieur, le "savoir savant » se distinguerait du « savoir de révélation » par la mise en place de procédures d'observation ou d'analyse (28-29). De l'autre côté, les "savoirs de croyance» découleraient des considérations que le sujet porte sur le fondement des événements et qui sont par la suite intériorisées en savoir (31). Au sein de ces "savoirs de croyance ", le rapport du sujet au monde semble important : « soit le sujet s'impose au monde pour le décrire (savoir d'expérience), soit pour porter des jugements (savoirs d'opinion)» (31).

5 Charaudeau observe ensuite plusieurs figures de vérité induites par le désir du sujet parlant d'être reconnu comme crédible. Emprunté au vocabulaire des Fragments du discours amoureux de Roland Barthes, le terme de «figures » est ici associé à celui de vérité. Les figures de vérité décrites par l'auteur sont liées à l'intentionnalité du sujet. Dépendantes du mode d'énonciation des types de savoirs précédemment décrits, elles peuvent selon l'auteur être tournées vers le monde (vérité factuelle et vérité scientifique), vers le locuteur lui-même comme sujet ou discours (vérité doctrinale et vérité conviction) ou enfin, vers l'autre, l'interlocuteur (vérité sincérité et vérité consensus). L'ouvrage se signale ainsi par son souci de la cohérence des éléments présentés : avant de passer à l'étude des conditions de l'acte de communication dans lesquelles peuvent se manifester les figures de la vérité dans différents domaines de pratique sociale, il prend soin de relier les figures de vérité aux types de savoirs tout en présentant de nombreux exemples.

6 Cette première partie de l'ouvrage constituée par la présentation des conditions d'analyse $d u$ discours manipulatoire se poursuit par l'étude des outils de travestissement de la vérité. Charaudeau commence par rappeler que l'affirmation d'une vérité, abordée de manière langagière, suppose la possibilité pour celle-ci d'être niée. Présupposant la vérité tout en ouvrant la possibilité d'une discussion, l'altération de la vérité est de fait examinée ici comme un acte de «négation de langue »: celle-ci " présuppose une existence car il faut concevoir pour nier » (51). En effet, la négation est perçue comme un acte à la fois interactif et intersubjectif car, selon l'auteur, elle dépend de tous les acteurs et toutes les circonstances de l'acte de parole. Après avoir étudié les divers mécanismes de la négativité, l'auteur propose une intéressante distinction des figures de la négation qui traversent la vérité d'un point de vue linguistique. Comme il le rappelle, ces concepts souvent confondus se signalent pourtant par des intentions, des conditions, des effets et des paradoxes qui leur sont propres. Ainsi, il passe en revue les diverses figures de la négation que sont le mensonge (59), la dénégation (62), la mauvaise foi (65) et l'imposture (70) avant de conclure cette première partie par des réflexions sur la nature et les moyens du débat public.

7 S'appuyant sur les conditions d'un examen langagier de la manipulation (Première Partie), la suite de l'ouvrage s'attache naturellement à procéder à son analyse. L'auteur 
se penche tout d'abord sur les problèmes d'identification des cas de manipulation et les distingue des actes de persuasion (86). Cela le conduit ensuite à présenter, fort pertinemment, les spécificités du discours manipulatoire. Abordant celui-ci comme un ensemble de discours fragmentés (du fait de sa difficile chronologie dans la chaîne de corrélation) et destiné à un public hétérogène, Charaudeau pointe le maquillage intentionnel et les effets de tromperie suscités par le locuteur manipulateur au moyen de plusieurs outils. Qu'il s'agisse de l'utilisation des différentes figures de négation, des rôles incarnés, ou encore des procédés discursifs employés de manière éclectique par le locuteur, l'auteur montre que la manipulation repose sur différentes stratégies d'incitation. Si elles visent comme toute persuasion «à amener autrui à faire, dire, penser, ce que le sujet parlant voudrait qu'il fasse, dise ou pense », elles se distinguent " par un maquillage intentionnel et un effet de tromperie » (86). Pour arriver à ses fins, le manipulateur adopte divers rôles, comme le conseilleur, le guide (éventuellement charismatique), l'animateur. Il utilise divers procédés discursifs comme l'exagération, la généralisation, l'amalgame, les arguments de cause fallacieux, l'humour... Ils reposent sur des stratégies d'incitation positive (créer la confiance ou l'amitié par exemple) ou négatives (pratique de la dénonciation, l'accusation, la stigmatisation, etc. pour provoquer protestation, revendication, révolte). Elles apparaissent dans différentes attitudes manipulatoires en fonction de la position du sujet manipulateur (88) : attitude volontaire à effet de suggestion et de consentement; attitude volontaire à effet de tromperie; attitude non intentionnelle).

8 Le passage en revue de celles-ci lui permet d'envisager avec précision des genres associés à la manipulation comme la publicité, le mensonge en politique (stratégie du déni, du secret...), les discours de la peur, la rumeur (exemple d'attitude non intentionnelle), et bien d'autres. Ainsi, par exemple, si elles relèvent bien toutes les deux d'une manipulation volontaire et qu'elles sont, par conséquent, souvent assimilées, la publicité se distingue de la propagande tactique en ce que, jouant davantage sur le désir des consommateurs, elle n'a pas besoin de se présenter dans une position d'autorité. Achevant cette seconde partie, l'auteur prend bien soin de nuancer l'approche catégorisante de certaines analyses des discours en rappelant que ceux-ci ne sont pas également manipulatoires (115).

Avant de conclure, le livre interroge les cadres habituels de réflexion du concept de post-vérité en proposant une analyse langagière de cette nontion à la mode. À ce titre, Charaudeau rappelle les écueils que peut susciter l'utilisation abusive de ce terme tant en raison des généralisations que des oublis de l'histoire qu'il autorise. Portant davantage son attention sur la façon dont est énoncé ce qui semble une vérité, l'auteur entend montrer que l'essentiel de la problématique de la post-vérité ne se situe pas dans l'influence décroissante ou non des faits objectifs sur l'opinion publique, mais plutôt dans « la représentation que l'acte de langage donne de ces faits, selon qu'il fait appel à un savoir savant ou à un savoir d'expérience » (124). Dans cette optique, les fake-news et autres infox sont considérées comme des contre-vérités (126); la perspective discursive y distingue entre ceux qui procèdent de la négation des faits (négationnisme) et ceux qui découlent d'une invention de ceux-ci (révisionnisme et complotisme). Cette transformation des messages peut s'effectuer selon différents motifs en vue de différents impacts (133), mais n'est pas sans poser des problèmes d'enjeux démocratiques, notamment aux médias chez qui Charaudeau relève différents manquements, effets pervers et contradictions. Face à ce qu'il qualifie de véritable triomphe de la négation, l'auteur finit par plaider pour une forme d'équilibre entre 
passion et raison qui, selon lui, devrait inciter les médias à s'interroger « sur leur façon d'informer sans dénégation" (146). À l'heure où de nombreuses polémiques se déclenchent sur les réseaux sociaux ${ }^{1}$, nul doute que ces éclairages et ces propositions soient plus que jamais d'actualité.

Petit concentré de réflexions et d'outils conceptuels importants pour envisager le discours manipulatoire et la post-vérité, le livre de Patrick Charaudeau nous propose un approfondissement enrichissant et précieux de ces sujets ô combien sensibles.

\section{BIBLIOGRAPHY}

Bigot, Laurent. 2019. Fact-checking vs. fake news. Vérifier pour mieux informer (Paris : INA Éditions)

Bronner, Gérald. 2021. Apocalypse cognitive (Paris : PUF)

Charaudeau Patrick. 2007. «Les stéréotypes, c'est bien. Les imaginaires, c'est mieux », Boyer, Henri (éd.). Stéréotypage, stéréotypes : fonctionnements ordinaires et mises en scène, vol. 4 (Paris :

L'Harmattan), 49 - 63

Ellul, Jacques. 1990. Propagandes (Paris : Economica)

Ferraris, Mauricio. 2019. Postvérité et autres énigmes (Paris : PUF)

Huyghe, François-Bernard. 2016. La désinformation. Les armes du faux (Paris : Colin)

Klein, Étienne. 2020. Le Goût du vrai (Paris : Gallimard)

Williams, Bernard. 2006, Vérité et véracité (Paris : Gallimard)

\section{NOTES}

1. Et notamment ces dernières semaines avec le dessin de Xavier Gorce lui-même dans le journal Le Monde.

\section{AUTHORS}

\section{SÉBASTIEN CHONAVEY}

Université libre de Bruxelles 